\title{
Force-Controlled Formation of
} Dynamic Nanopores for SingleBiomolecule Sensing and SingleCell Secretomics

\section{Journal Article}

Author(s):

Schlotter, Tilman (D); Weaver, Sean; Forró, Csaba; Momotenko, Dmitry; Vörös, Janos (D); Zambelli, Tomaso; Aramesh, Morteza (D)

Publication date:

2020-10-27

Permanent link:

https://doi.org/10.3929/ethz-b-000450209

Rights / license:

$\underline{\text { In Copyright - Non-Commercial Use Permitted }}$

Originally published in:

ACS Nano 14(10), https://doi.org/10.1021/acsnano.0c04281

Funding acknowledgement:

174217 - Single Entities at High Magnification: Mapping, Measuring and Manipulating Nanoparticles (SNF) 


\section{Force-controlled formation of dynamic nanopores for}

\section{single-biomolecule sensing and single-cell secretomics}

Tilman Schlotter†, Sean Weaver†, Csaba Forró†\|, Dmitry Momotenko†, János Vörös†, Tomaso

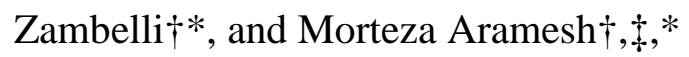

† Laboratory of Biosensors and Bioelectronics, Institute for Biomedical Engineering, ETH Zürich, 8092

Zürich, Switzerland

\| Department of Chemistry, Stanford University, Stanford, CA 94305, USA

‡ Laboratory of Applied Mechanobiology, Department for Health Sciences and Technology,

ETH Zürich, 8093 Zürich, Switzerland 


\begin{abstract}
Nanopore sensing of single nucleotides has emerged as a promising single-molecule technology for DNA sequencing and proteomics. Despite the conceptual simplicity of nanopores, adoption of this technology for practical applications has been limited by a lack of pore size adjustability and inability to perform long-term recordings in complex solutions. Here we introduce a method for fast and precise ondemand formation of a nanopore with controllable size between 2-20 nm through force-controlled adjustment of the nanospace formed between the opening of a microfluidic device (made of silicon nitride) and a soft polymeric substrate. The introduced nanopore system enables stable measurements at arbitrary locations. By accurately positioning the nanopore in the proximity of single neurons and continuously recording single molecule translations over several hours we have demonstrated this is a powerful approach for single cell proteomics and secretomics.
\end{abstract}

Keywords: Dynamic nanopores, biomolecular sensors, AFM-integrated nanopores, nanoconfinement of biomolecules, single-cell proteomics. 
Nanopores are fundamental to life since they enable cell regulation and many cell-to-cell interactions. ${ }^{1}$ The translocation of an object such as an ion or a molecule is enabled by the constriction of the pore, allowing passage only to objects with specific physical or chemical properties. ${ }^{1}$ Solid-state and biological nanopore systems have been developed over the last two decades for various applications ranging from water desalination ${ }^{2}$ or energy generation ${ }^{3}$ to biomolecular sensing and diagnostics. ${ }^{4}$ Biological nanopores, such as $\alpha$-haemolysin, have enabled DNA sequencing, ${ }^{5}$ and paved the way towards commercially available low-cost DNA sequencing devices. ${ }^{6,7}$ Other applications include the determination of singlemolecule kinetics ${ }^{8}$ or the directional control of a molecular hopper. ${ }^{9}$ On the other hand, solid-state nanopores (such as silicon-nitride, ${ }^{10}$ graphene, ${ }^{11,12} \mathrm{MoS}_{2}{ }^{13}$ or $\mathrm{WS}_{2},{ }^{14}$ and glass pipette ${ }^{15,16}$ pores) have shown promise for protein sensing ${ }^{17}$ since they can operate under a wider range of environmental conditions, including high electrical bias potentials, ${ }^{18}$ high temperatures ${ }^{19}$ and function in the presence of denaturants like sodium dodecyl sulfate (SDS). ${ }^{20}$

Despite the conceptual simplicity of nanopores, pore-clogging by proteins or other molecules and nanopore degradation often remain a serious bottleneck for long-term measurements. ${ }^{21-23}$ Moreover, to measure multiple proteins of different dimensions or charge, nanopores with adjustable openings are needed. Both biological and solid-state nanopores have fixed aperture sizes which cannot be changed on demand. Another major challenge is to increase the temporal resolution for detection of the translocating molecules. ${ }^{24}$ One possibility to improve temporal resolution is to increase the sampling rate of the electrical signal, which comes at a cost of higher electrical noise. ${ }^{25}$ Hence, recent attempts focus on slowing-down the translocation velocity of the biomolecule, for example by introducing a physical barrier for molecular diffusion, ${ }^{26-29}$ controlling electrostatic forces, ${ }^{30}$ pressure, ${ }^{31}$ or chemical modification of the pore's surface. ${ }^{32}$ 
In this work, we propose a method to form a force-controlled nanopore (FCNP) by modifying the aperture of a rigid micropore mounted on an Atomic Force Microscope (AFM) when bringing it in contact with a soft substrate. The proposed system creates variable sized nanopore able to take measurements over the course of several hours. We show the technique enables localized detection and characterization of nanoparticles, DNA, proteins and peptides. Further, we demonstrate long-term monitoring of single-cell neural activity by measuring the size-specific time resolved secretome. 


\section{Results and Discussion}

\section{Formation of a force-controlled nanopore at the interface of a rigid, micro-structured and soft, polymer surface}

A force-controlled nanopore (FCNP) was formed at the interface between a soft polydimethylsiloxane (PDMS) substrate and the aperture of a $\mathrm{Si}_{3} \mathrm{~N}_{4}$ micro-channeled AFM cantilever ${ }^{33}$ creating a nanoconfined

area (Figure 1a). The cantilever, with a nominal spring constant of $2 \mathrm{~N} / \mathrm{m}$, has a $10 \times 10 \times 7 \mu^{3}$ pyramid at the extremity with an aperture of $300 \mathrm{~nm}$ diameter at its apex. The aperture edge is not on a planar surface, but it is characterized by four sharp corners that form arches with a maximum height of 20-50 nm along the perimeter (see Figure 1b). The ionic current through the aperture was measured using two $\mathrm{Ag} / \mathrm{AgCl}$ electrodes - a reference electrode in solution and a working electrode inside the cantilever reservoir. The cantilever was mounted on an AFM head (with an angle $\alpha$ of $\sim 11^{\circ}$ ), allowing control of the force between the cantilever tip and the substrate. During the approach, movement of the cantilever was monitored through an optical prism (to reflect light $90^{\circ}$ into the optical microscope). The highest force applied was $2 \mu \mathrm{N}$, leading to a maximum deflection angle of $1.15^{\circ}$ along the $40 \mu \mathrm{m}$ cantilever with a spring constant of $2 \mathrm{~N} / \mathrm{m}$. Therefore, a constant angle of $\sim 11^{\circ}$ (the mounting angle) is assumed between the apex of the cantilever and the surface of the PDMS substrate at all forces (see Figure 1c-e). The force between the cantilever and the substrate results in an indentation of the probe apex into the substrate. The indentation depth is defined as the difference between piezo position from the contact point and the deflection (bending) of the cantilever. The displacement-force curves of a typical indentation of the cantilever into a PDMS layer with Young's modulus of 2 MPa are shown in Figure 1f. The indentation showed a linear behavior with increasing force and dependency on the substrate stiffness (Figure S8 and Table S1). 
For the electrochemical characterization of the nanopore at different applied forces, I-V curves were recorded in $2 \mathrm{M} \mathrm{KCl}$ and $150 \mathrm{mM}$ phosphate buffered saline (PBS) at $\mathrm{pH} 7.4$ (Figure 2a and b). The pore exhibited a linear resistive characteristic when the apex was far from the substrate, whereas the curve deviated significantly from the linear behavior when the apex was in contact with the substrate. The resistance across the pore increased with the applied force, suggesting the nanopore size decreased. Rectification of the ionic current occurred at applied forces exceeding $0.1 \mu \mathrm{N}$, indicating the surface charge both of the pyramidal silicon nitride probe and the spin-coated PDMS substrate strongly influence the measured conductivity due to the influence of electrical double layer in confined spaces. ${ }^{34-37}$ At lower salt concentrations (Figure 2b) the double layer effect is stronger due to the increased thickness of the double layer.

The nanopore formed instantaneously as the AFM tip came into contact with the substrate, as it is indicated by the instant change in the ionic current upon contact with the surface (Figure 2c). In these experiments the cantilever approached the surface with a constant speed until a set point force was reached. For comparison with the behavior on soft substrates, distance-current curves were obtained when the AFM cantilever approached a glass substrate (stiffness in the GPa range). The resistance drop was less than 0.1 $\mathrm{M} \Omega$, not sufficient to form a FCNP (Figure S9a). Therefore, we concluded that contact between a rigid and a soft material is essential for the formation of a FCNP. The current stabilizes after the contact; however, with a drift proportional to the force. The drift decayed after a few minutes (Figures S11b and S12b) and subsequently disappeared from the measured data.

A finite element method simulation was used to estimate the nanopore size as a function of the maximum gap height between the cantilever and the substrate (Figure S5 and S6). The simulation was carried out for two scenarios (Figure 2d): a) a flat indentation of the cantilever and b) an indentation at the mounting angle of $11^{\circ}$. The obtained resistance values from scenario b) corroborated the experimental results, 
supporting our hypothesis that the contact angle between the apex and the substrate remains constant as shown before. The simulations further indicated that control of the nanopore with single nanometer precision is possible. However, the simulation did not include surface charge effects which play a crucial role in the conductive properties of the FCNP. Therefore, to validate our estimation of the size of the FCNP, translocation of different nanoparticles and DNA molecules through the adjustable nanopore was investigated - always with a positive bias applied between the two electrodes.

\section{Nanopore calibration with nanoparticle and DNA translocations}

Translocation of polystyrene nanoparticles with a mean diameter of $20 \mathrm{~nm}$ through the nanopore was recorded. The particles were functionalized with carboxyl (-COO$)$ groups resulting in a negative net charge. While the translocation events were observed for force set point values of $5 \mathrm{nN}$ and $10 \mathrm{nN}$, the pore was too small for nanoparticles translocation at a set point higher than $50 \mathrm{nN}$ (Figure S14) - thus, the biggest separation between PDMS and apex (i.e. nanopore dimension) is estimated to be less than 20 $\mathrm{nm}$ at set points $>50 \mathrm{nN}$.

To estimate the nanopore size at forces up to $2 \mu \mathrm{N}$, the translocation of carboxylated gold nanoparticles with a mean diameter of $6 \mathrm{~nm}$ was investigated (Figure 3a). Figure 3b-d show examples of baseline subtracted current transients induced by gold nanoparticle translocations at different set points. For a set point value of $0.01 \mu \mathrm{N}$, translocation events could not be detected due to the low signal to noise ratio (SNR). However, at higher forces (e.g. $0.08 \mu \mathrm{N})$ the higher confinement of a nanopore enabled higher SNR for nanoparticle translocations. At $2 \mu \mathrm{N}$ the NPs were "squeezed" between the PDMS and the apex edges resulting in translocation times of several seconds (Figure 3e) which corroborates the simulations, further demonstrating nanometer tuning of the dimensions of the FCNP. 
The translocation of negatively charged nanoparticles yielded an increase in the ionic current in our system while translocation of biomolecules through a free-standing nanopore generally results in a resistive pulse. Here, dominance of charge screening over the spatial blockade leads to current enhancement. For example, at low solution conentrations, where the thickness of the double layer increases, the charge screening via counter-ions becomes significant and therefore a current enhancement occurs upon DNA translocation. ${ }^{38}$ Similarly, when two charged surfaces are brought in close proximity, their double layers interfere, creating a charge sensitive region. ${ }^{39}$

In addition to the applied force, the electric potential can be adjusted to characterize the translocating analytes. For this purpose, double-stranded DNA (dsDNA) was selected as a benchmark analyte since it is often used in many single biomolecule nanopore experiments ${ }^{40-42}$ due to the small dimension $(\sim 2 \mathrm{~nm}$ thick), high negative charge at neutral $\mathrm{pH}$, mechanical stability and the lack of secondary or tertiary structures. The voltage-dependent translocation of DNA molecules was investigated at a constant force of $1 \mu \mathrm{N}$. dsDNA molecules with 2000 base pairs were added to the bath solution ( $2 \mathrm{M} \mathrm{KCl}, \mathrm{pH} \sim 8$ ) and their translocation events recorded (Figure 3f). The mean peak current of the DNA translocations strictly increased with a broadened distribution (Figure 3g), while the translocation time decreased (Figure 3h), upon ramping the applied potential from 0.1 to $0.5 \mathrm{~V}$. The translocation frequency was increased and the translocation time was broadened further, by increasing the voltage to $2 \mathrm{~V}$, but the variance of peak shape in the current transients was increased at voltages between 1-2 V (Figure 3i-k). This can be attributed to the translocation of DNA with certain conformations that could translocate through the nanopore only at a higher driving voltage.

Classification of the peaks by their shape is a method to distinguish various conformations. Here, we used the Principle Component Analysis (PCA) with 10 principle components and subsequent clustering (Figure S16) to classify the translocation events, as shown in Figure 3l-n. Translocation events at lower 
voltages are characterized with a single sharp profile. At $1 \mathrm{~V}$, the PCA does not distinguish clearly between two or three different shapes, which indicates that DNA may enter the FCNP with many different conformations at this voltage. This observation is supported by the distribution profile in the density plots (Figure 3o-q), which highlights two distinct current peaks of 16 and $22 \mathrm{nA}$ at $0.1 \mathrm{~V}$. The distribution profiles are even narrower for translocations at a potential of $0.25 \mathrm{~V}$. However, at $1 \mathrm{~V}$ the distribution profile is broader, where one can see that the translocation time correlates with the magnitude of the current change (e.g. short translocation times, $2 \mathrm{~ms}$, correlate to small peak currents and longer translocation times, 4 ms, yield larger peak currents).

\section{Force-dependent translocation measurements of peptide Dynorphin A and BSA protein}

Next we evaluated the pore size adjustability to enable measurements of small biomolecules, such as single peptides lacking secondary or tertiary structure. Porcine dynophin A (Dyn), an opioid neuropeptide that is widely distributed in the central nervous system and regulates the neural activity, ${ }^{43}$ has 17 residues, a net charge of $+4 e$ at $\mathrm{pH} 7.0$, and $2.15 \mathrm{kDa}$ molecular weight. Dyn translocation through the nanopore was measured in pure PBS, where the cantilever reservoir was filled with Dyn and set to a negative potential. Figure 4a-c show current traces with an applied electrical potential of $1 \mathrm{~V}$ at $0.1,0.5$, and 1.0 $\mu \mathrm{N}$ set points. The translocation time of the peptide varied from 5 to around 20 milliseconds (Figure 4de). The measured translocation times were by more than three orders of magnitude longer than those predicted by molecular dynamic (MD) simulations. For example, MD simulations predicts that a 48 residues-long peptide translocates through a $2.2 \mathrm{~nm} \mathrm{MoS} 2$ nanopore within $0.3 \mu \mathrm{s}^{44}$ The observed difference is most probably due to the confinement effects by the FCNP, which is not observable in conventional nanopore systems. 
To further understand the conformational states of the peptide during the translocation, principle and independent component analyses (PCA and ICA) were performed. The normalized events with three principle components (Figure S17) show that the current transients due to fast translocation of Dyn had a pulse shape that appeared as a plateau at $1 \mathrm{~V}$ applied potential. At a lower potential of $0.75 \mathrm{~V}$ and at a higher force of $1.3 \mu \mathrm{N}$ an additional translocation confirmation appeared, distinguishable by its current transient shape. These conformations likely arose because of the increased interaction between the peptide and the nanopore at the lower potentials, which resulted in longer translocation times of more than $25 \mathrm{~ms}$ (Figure S18f). These translocations were not observed at higher voltages (i.e. increased electrostatic forces on the peptide), suggesting that the peptide-pore interaction was reduced at higher potentials (Figures S18g-i).

Laminin (810 kDa), which promotes the differentiation and growth of neurons, ${ }^{45}$ was too big to pass through the FCNP (Figure S19). Hence, to compare the translocation times in this study with those of other reports, translocation of the smaller and well-studied bovine serum albumin (BSA) was investigated. BSA has a molecular weight of $66.5 \mathrm{kDa}$ and an isoelectric point of 5.4, having an estimated charge of $9 e$ at $\mathrm{pH} 7.4 .^{46}$ The primary structure consists of 583 residues that give BSA the cell dimensions of $21.6 \times$ $4.5 \times 14.2 \mathrm{~nm}^{3} 47$ (Figure 4 3D-structure). The translocations were measured at a positive potential (1 V) and at 0.9-1.25 $\mu \mathrm{N}$ set point force (Figure 4f-i). BSA translocations were identified by a drop in the current (contrary to the previous measurements with DNA, peptide and nanoparticles - e.g. see Figure 3), suggesting that the pore blockade by BSA contributes more than charge screening in the recorded currents. Through conventional solid-state nanopores translocation times below $2 \mathrm{~ms}^{48,49}$ are reported. Translocation times with the FCNP were in the order of 250 to 500 ms (Figure 4j-k), i.e. two orders of magnitude longer. Similar to our previous measurements, increasing the set point first led to an increased variance in the peak shape classes (e.g. appearance of multiple minima), and subsequently by increasing 
the set point to still higher values (e.g. $1.25 \mu \mathrm{N}$, Figure 4h), the pore size became so small that no more translocations were detected. In the case of BSA, the stronger interaction with the pore at higher forces could additionally result in a conformational change of the tertiary and possibly secondary structure of the protein which is concluded from additional minima in the translocation signal.

\section{Monitoring neural secretome at the single-cell level}

The pore size adjustability in FCNP enabled size-specific, localized recordings next to the single neural

cells (Figure 5a). The cells were cultured on a glass slide coated with PDMS, as reported previously. ${ }^{50}$ The AFM tip can be positioned close to the cells with a high accuracy. ${ }^{51}$ Bright-field and fluorescent (GFP) images of the cantilever with the living neural cells after six days in vitro culture (DIV 6) are shown in Figure 5b-c.

Secretion of biomolecules from single neurons was monitored by positioning the AFM tip next to the individual cells. Each cell dynamically secrets a wide range of biomolecules that possess a range of charges and molecular weights. ${ }^{52}$ Indeed, a wide range of translocations were detected at $0.2 \mu \mathrm{N}$ set point and $1 \mathrm{~V}$ applied potential (Figure 5g). Due to the small size of the pore $(<20 \mathrm{~nm})$ at this set point, it is reasonable to assume that neural exosomes, with a size ranging from $50-100 \mathrm{~nm},{ }^{53}$ do not translocate through the pore (as it was shown that $20 \mathrm{~nm}$ nanoparticles cannot enter the FCNP at > $50 \mathrm{nN}$ set point).

To enable size-controlled detection of the secreted molecules, the current recordings were performed at different force set points. The pore size reduced with the set point, resulting in a higher SNR and longer translocation times in the observed events (Figure 5d-f). To detect only the larger biomolecules, such as those similar to BSA (i.e. $21.6 \times 4.5 \times 14.2 \mathrm{~nm}^{3}$ ), the pore was adjusted to $1 \mu \mathrm{N}$ set point and $1 \mathrm{~V}$ potential was applied. The translocation events are shown in Figure 5h, where a single cluster of events is observed with a mean translocation time of $500 \mathrm{~ms}$ and a mean current change of $1.9 \mathrm{nA}$. As shown by 
the measurements of dynorphin, FCNP exhibited bias towards the short biomolecules with no or little tertiary structure at higher set points, e.g. at $2.0 \mu \mathrm{N}$. Similarly, when the set point was increased to $2.0 \mu \mathrm{N}$ during the neural recording, events with a translocation time below 100 ms appeared with higher frequency (Figure 5i). Additionally, the maximum translocation time increased with increasing set point suggesting a strong interaction between the biomolecules and the pore walls in this range (Figure 5k). Interestingly, at this set point we observed that the released “peptide-like” signals by a neuron appear in "trains”, where successive translocations are followed by intervals with lower translocation frequencies (Figure 5f). In Figure S20 current traces at even higher forces are shown which resolved the events with more details. These spike trains were not observed during the measurement of macromolecules at lower forces, indicating that these events are specific to the small size ('peptide-like”) molecules. These results suggest that FCNP is a powerful tool in monitoring the size-specific neural secretome at the single-cell level.

To evaluate the suitability of the FCNP for long-term cellular recordings, different measurements were conducted over 5 to $\sim 7$ h, continuously (Figure S21). The corresponding force-dependent measurements are shown in Figures S22 and S23. While clogging is an issue in most of the nanopore systems, no permanent clogging was observed in our long-term cell recordings. The activity of single neurons at DIV 6 was monitored over the course of $340 \mathrm{~min}$, at the constant potential of $2 \mathrm{~V}$ and the set point force of 1.0 $\mu \mathrm{N}$ (secretome maps Figure 5l-m). Dimensionality reduction of all recorded events was performed with UMAP, ${ }^{54}$ an algorithm that recently was introduced and has already been used to visualize highdimensional data of single-cells. ${ }^{55}$ The evolutionary secretion of proteins from a single neuron was resolved by UMAP, showing clockwise clustering (Figure 5m and Figure S24). This demonstrates the unique capability of the FCNP as a tool for time-resolved recording of the secretome of single-cells. This method could be combined with mass spectrometry to determine the specific type of secreted proteins/peptides. Such measurements can potentially show differences of various cells and indicate the 
evolutionary life of single-cells, providing a tool for biological disciplines that enables studying developmental biology, secretome-based communication such as in neural/immunological synapses, as well as monitoring antibody secretion from immune system cells for drug screening/discovery. 


\section{Conclusion}

In this work, we presented the concept of a force-controlled nanopore (FCNP) and demonstrated the ondemand control of a single nanopore with nanometer precision. The introduced platform relies on the contact between a rigid microstructure and a soft material, where the spacing is controlled by a piezo actuator. Nanopore calibration using nanoparticles and DNA fragments confirmed the simulations that the pore can be precisely controlled in a single nanometer range. It was furthermore shown that the strong interaction of the translocating molecules with the nanopore walls lead to an increase in translocation time of several orders of magnitude as compared to the conventional solid-state nanopores, allowing detection of very small biomolecules, such as peptides (2.15 kDa). Importantly, the FCNP is a clog-free system, which enables long-term measurements in complex environments, such as in proximity of a living cell. Measurements next to single neurons showed that the proposed FCNP system can be used to distinguish secreted products with different sizes. The system was also shown to be very robust and stable that allowed secretome monitoring in neuron cultures over several hours. In order to achieve functionality for singlecell proteomics, future applications may involve protein-specific surface functionalization of both the microstructure and the substrate, serial nanopores, protein-tailored nanostructures, and improved data processing. 

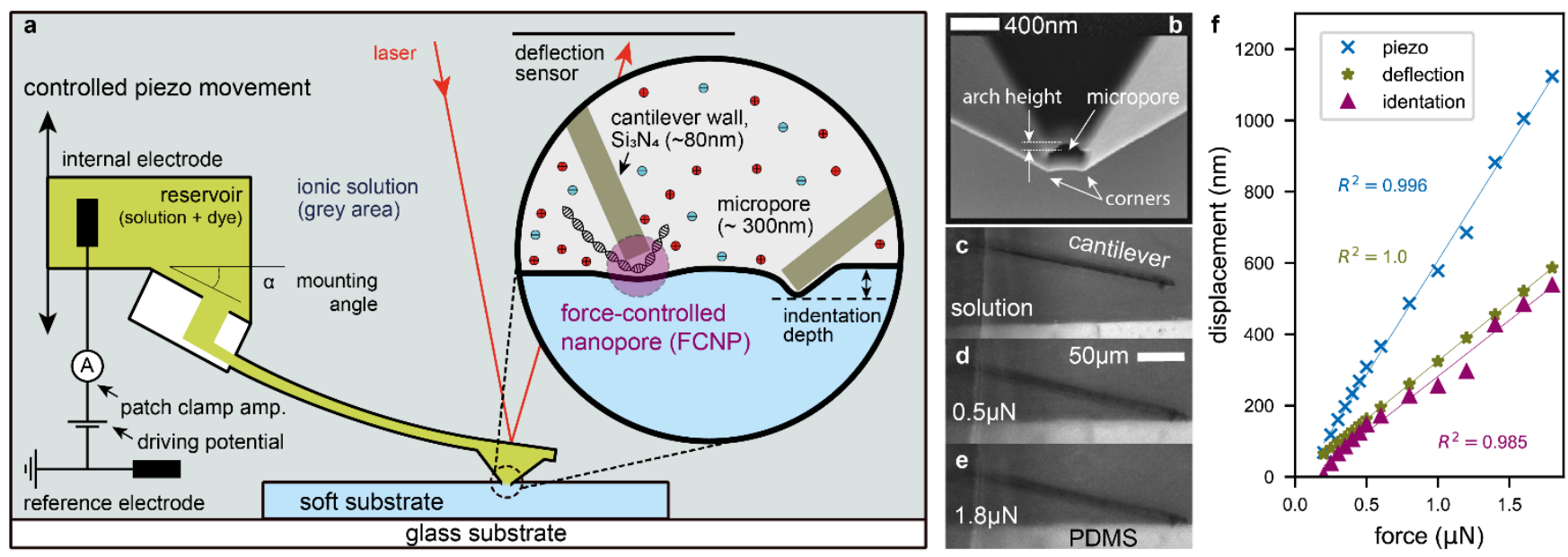

Figure 1. Formation of a force-controlled nanopore (FCNP) during indentation into PDMS. a, Schematic of the setup, built on an atomic force microscope (AFM) together with a micro-channeled cantilever. The adjustable nanoconfinement is formed at the interface between the cantilever edge (black) and the deformed PDMS substrate (blue). Schematic is not to scale b, SEM image of the apex of the used hollow AFM Si $\mathrm{N}_{4}$ cantilevers with an opening of $300 \mathrm{~nm}$. Nanopores are formed at the pore edges between the corners. c, Microscope images (through a $90^{\circ}$ optical prism) of the cantilever in solution at different set point forces, where $\mathbf{c}$ is the cantilever far from the surface, $\mathbf{d}$ the cantilever exerting a force of $0.5 \mu \mathrm{N}$ on the PDMS substrate, and e exerting a force of $1.8 \mu \mathrm{N}$ on the PDMS substrate. f, Indentation (displacement-force) curves on the PDMS substrate at forces between $0.2 \mu \mathrm{N}$ up to $1.8 \mu \mathrm{N}$ show linear indentation of the FCNP with increasing force. 

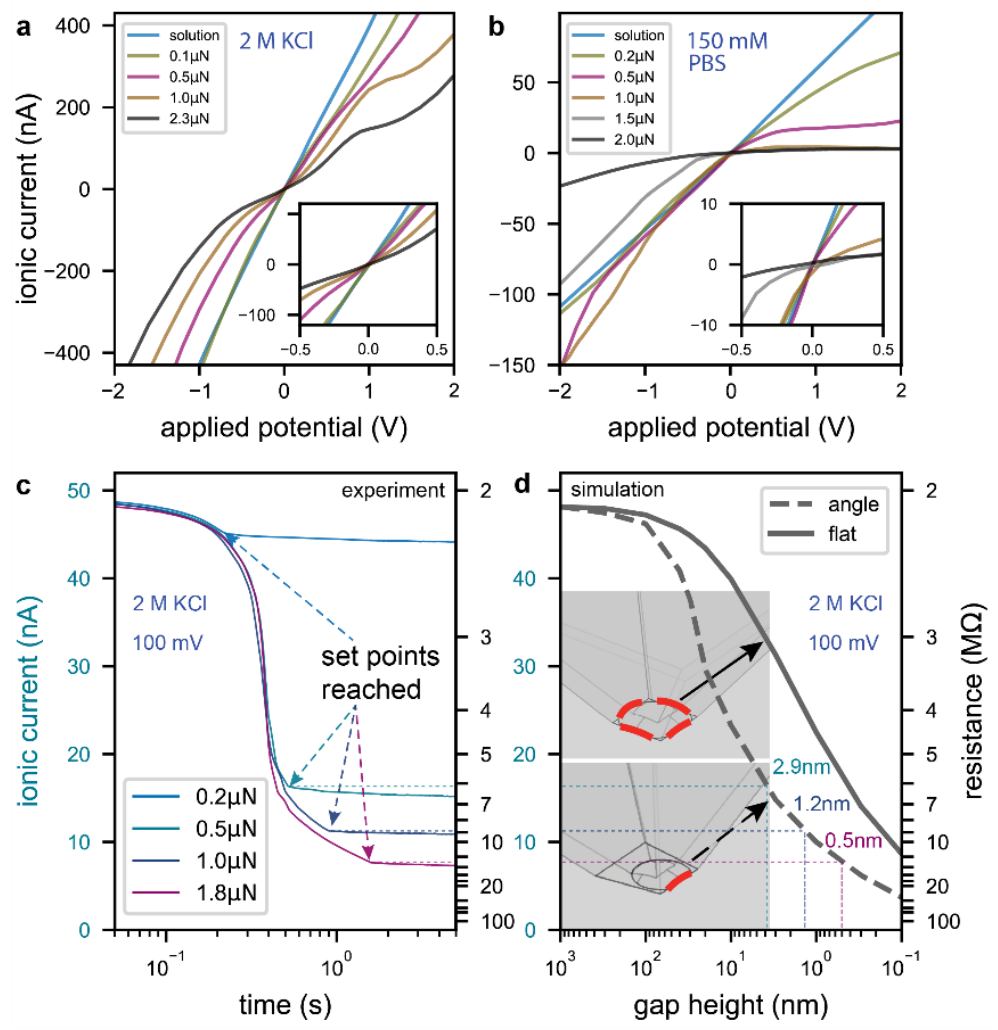

Figure 2. Characterization of the FCNP size at different forces. a, I-V curves in $2 \mathrm{M} \mathrm{KCl}$ solution from -2 to $+2 \mathrm{~V}$ (inset between -0.5 to $+0.5 \mathrm{~V})$. Saturation limit of amplifier reached at $\pm 420 \mathrm{nA}$. The conductance as a derivative of the ionic current with respect to the applied electric potential is shown at the bottom. b, I-V curves in $150 \mathrm{mM}$ PBS solution between -2 to $+2 \mathrm{~V}$ (inset between -0.5 to $+0.5 \mathrm{~V}$ ) and the corresponding conductance shown at the bottom; c, shows the ionic currents (left y-axis) and the corresponding resistance (right y-axis) during a constant-speed approach of the cantilever (100 mV applied potential); d, shows 3D COMSOL simulations of the ionic current through the nanopore at two different angles at an applied potential of $100 \mathrm{mV}$; Solid line represents $0^{\circ}$ angle configuration, where all four corners have the same distance to the substrate surface; Dashed line represents $11^{\circ}$ angle (corresponding to the mounting angle), where a nanopore is only formed at one side of the tip. Insets show geometry of a flat cantilever tip (top) and of a cantilever tip at an $11^{\circ}$ angle (bottom). Red lines correspond to cantilever edge where the nanopore is formed between the tip and the soft substrate. 2.9, 1.2, and $0.5 \mathrm{~nm}$ corresponds to the calculated gap height at the set points' current. 


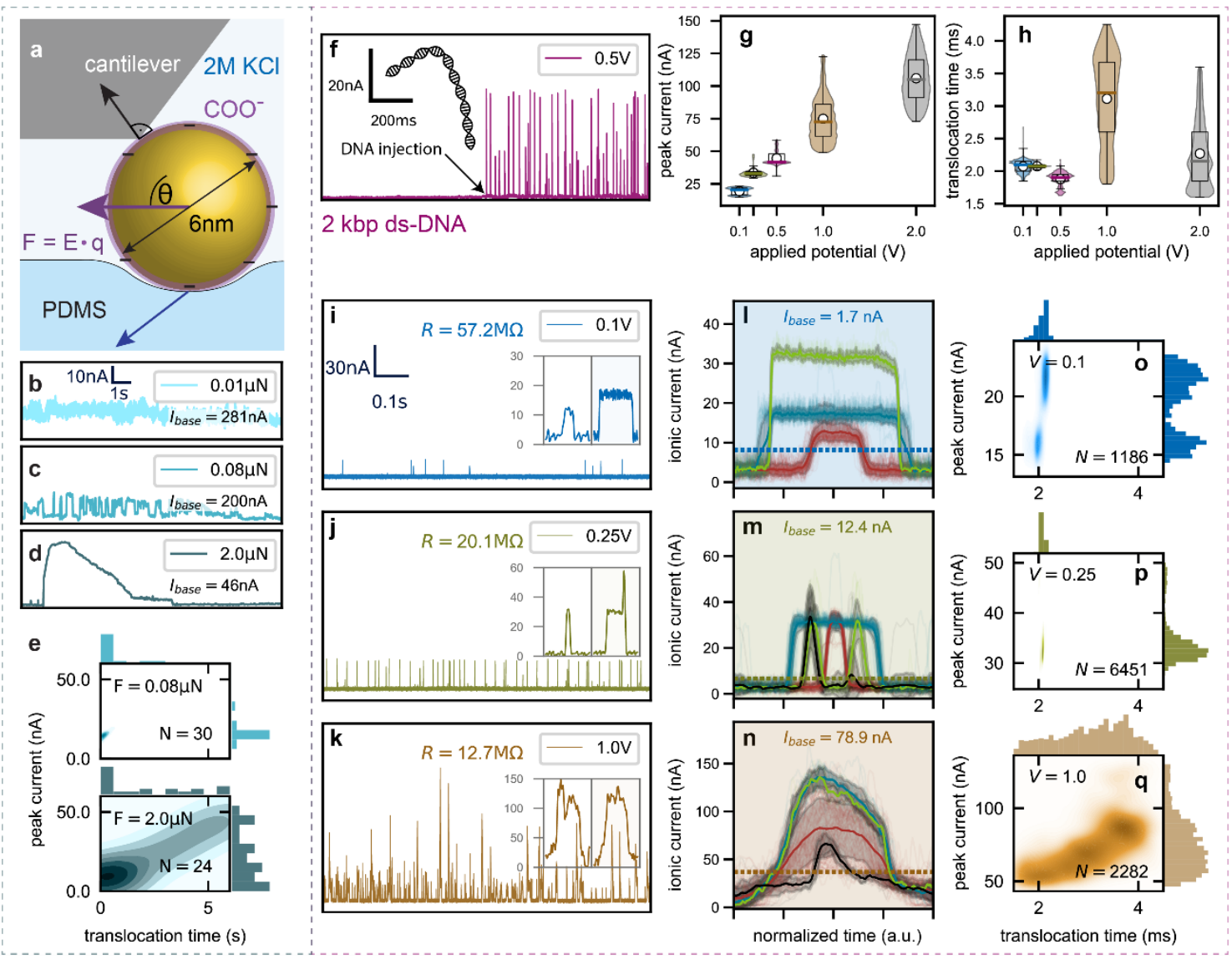

Figure 3. Nanopore calibration using nanoparticles and DNA. a shows an schematic illustration of the electrophoretic translocation of a negatively charged, $\mathrm{COO}^{-}$functionalized Au nanoparticle (with a diameter of $6 \mathrm{~nm}$ ) through the FCNP. The translocating particle exerts forces on the cantilever (black) and the PDMS substrate (blue) due to the mechanical confinement. b-d show current traces during nanoparticle translocations at an applied potential of $1 \mathrm{~V}$ and at three different force set points of $0.01,0.08$, and $2.0 \mu \mathrm{N}$ in $2 \mathrm{M} \mathrm{KCl}$. e, Density plots of translocations at 0.08 and $2.0 \mu \mathrm{N}$. f shows the change of ionic current measurements through the FCNP before and after injection of $2 \mathrm{kbp}$ ds-DNA fragments. The distribution of the peak currents and translocation times as a function of the applied electric potential are shown in $\mathbf{g}$ and $\mathbf{h}$, respectively. Violin and box-whisker plots show the data between $7^{\text {th }}$ and $93^{\text {rd }}$ percentile. The circle represents the mean value, the colored horizontal line indicates the median. The box shows the lower and upper quartiles. Subplots i-k show a section of the measured signals in $2 \mathrm{M} \mathrm{KCl}$ at $0.1,0.25$, and $1.0 \mathrm{~V}$ and share the same current and time scale. Inserts show two translocations with y-axis in nA and $\mathrm{x}$-axis not to scale. The results of a principle component analysis with three principle components from $\mathbf{I}$ to $\mathbf{n}$ show passage to distinct DNA conformation at low voltages but no well-defined principle components at an applied potential of $1 \mathrm{~V}$. Density plots of translocation time and peak current are presented in $\mathbf{0 - q}$. 
Dynorphine A (Dyn)
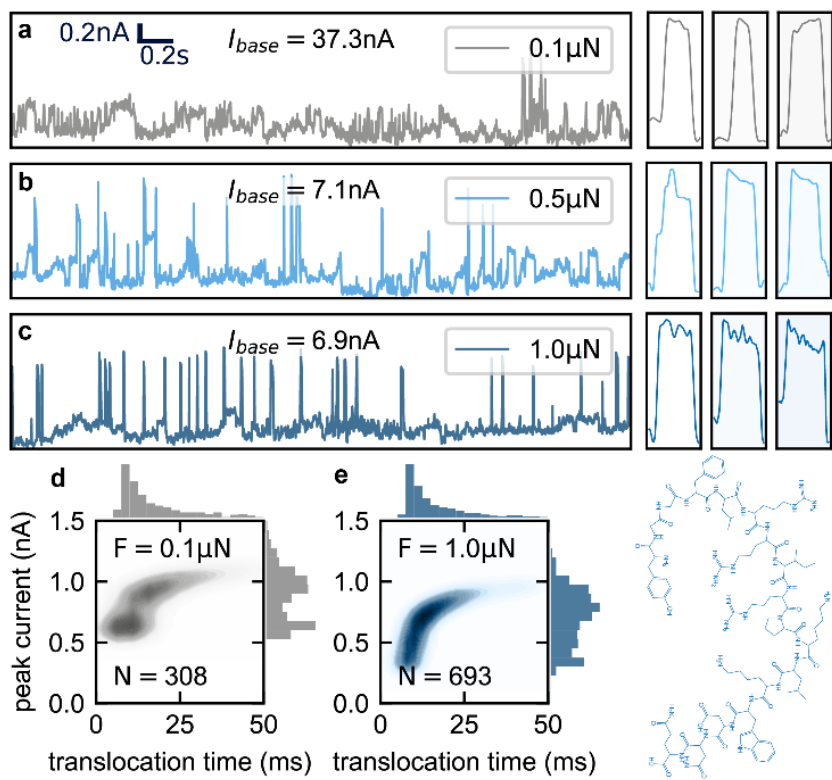

Bovine serum albumin (BSA)
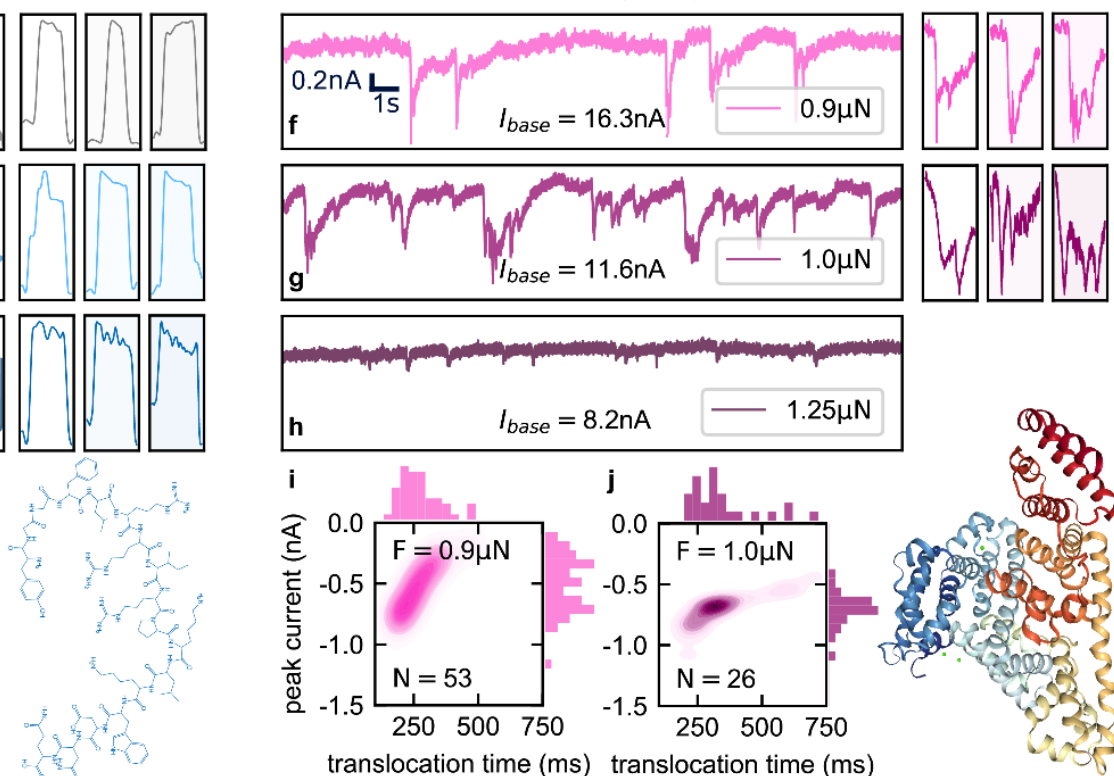

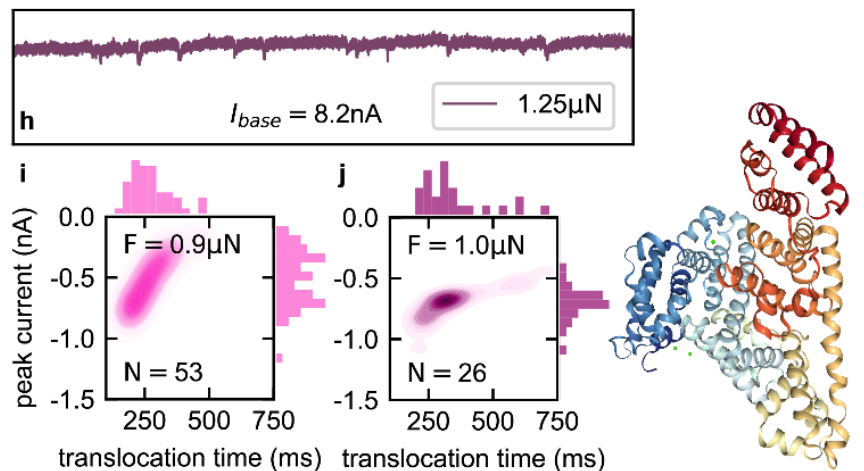

Figure 4. Translocation of Dynorphin A (Dyn) and Bovine serum albumin (BSA) at different forces. a-c, Current traces of Dyn (IEP: 11.3) filled into the cantilever reservoir in PBS solution at an applied potential of $1 \mathrm{~V}$ and at different forces of $0.1,0.5$, and $1.0 \mu \mathrm{N}$. All plots have the same scale. Three examples of translocation events are shown at different scales, next to each time trace. $\mathbf{d}$ and $\mathbf{e}$ show the corresponding density plots at 0.1 and $1.0 \mu \mathrm{N}$, respectively. In blue, the chemical structure of dynorphin is given. f-i show current traces of BSA (IEP: 5.4) in PBS solution outside the cantilever. All plots have the same scale. i shows that at a force of $1.25 \mu \mathrm{N}$, no BSA translocation through the pore was observed. Three examples of translocation events are shown at different scales, next to each time trace. $\mathbf{j}$ and $\mathbf{k}$ show the corresponding density plots at 0.9 and $1.1 \mu \mathrm{N}$. 3D structure of BSA (cell dimensions of $21.6 \times 4.5 \times 14.2 \mathrm{~nm}^{3}$ ) was taken from rcsb.org. 

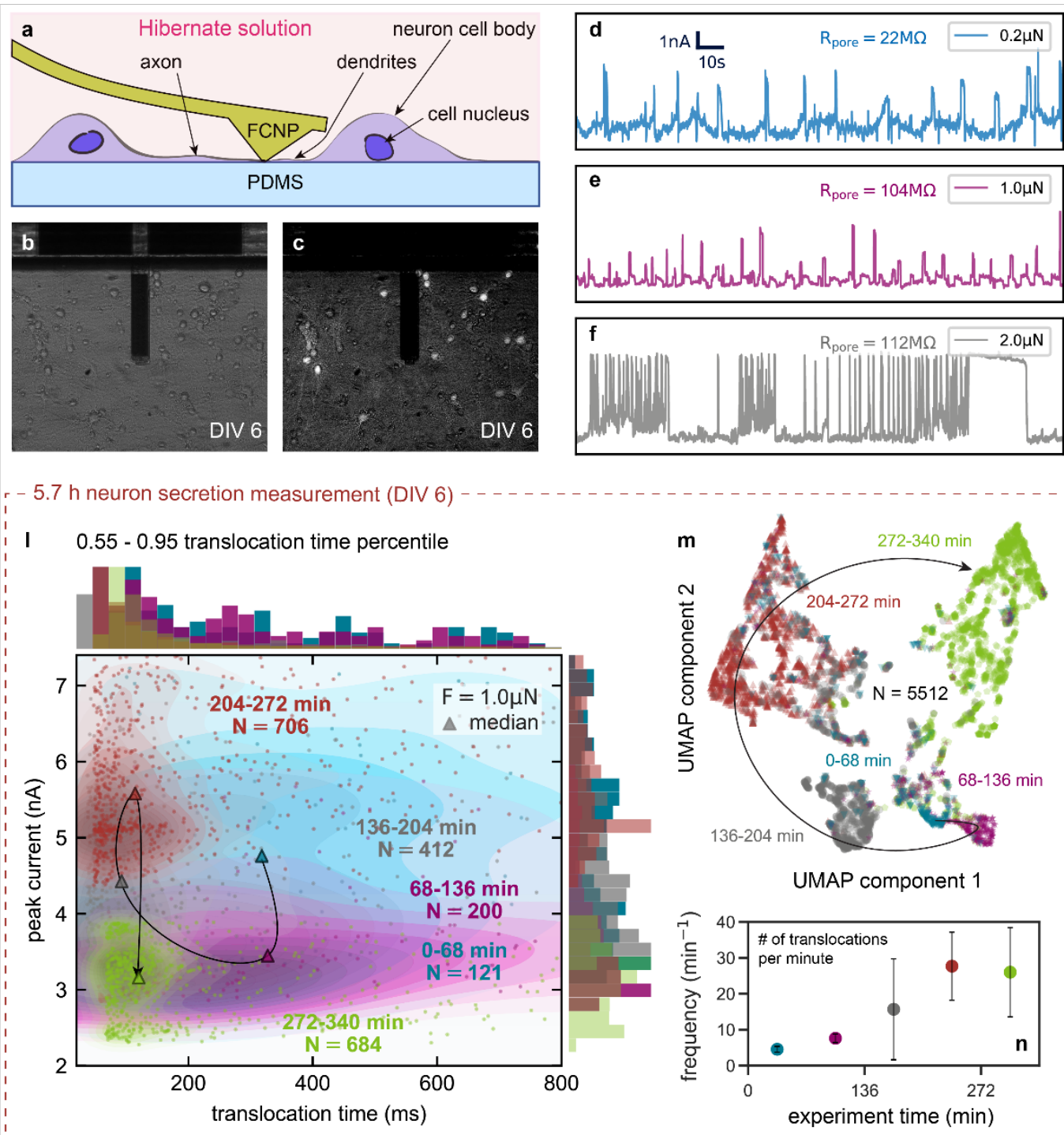
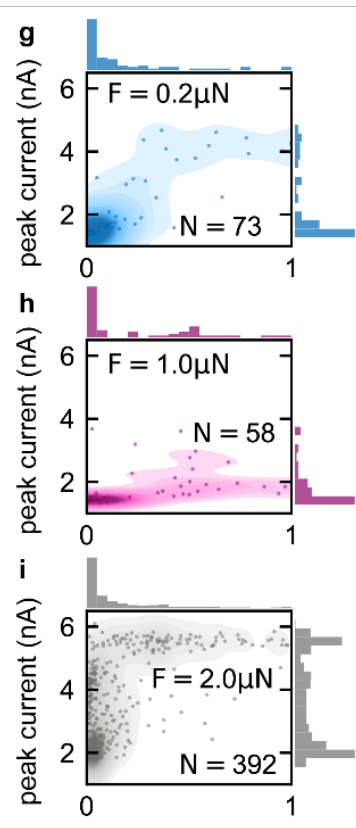

translocation time $(\mathrm{s})$
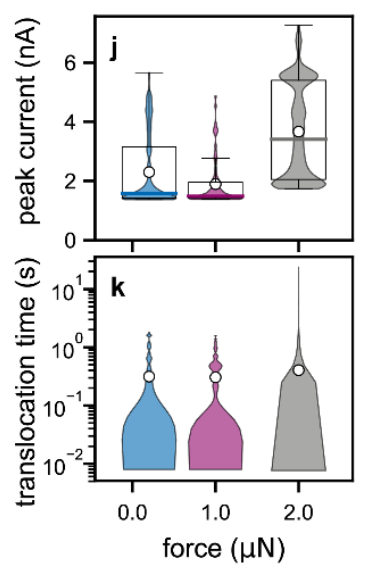

Figure 5. Size-specific and long-term detection of secretion from single-neurons. Neuron culture and AFM cantilever are imaged with bright field illumination a and $\mathbf{b}$ fluorescence illumination $\left(\mathrm{Ca}^{2+} \mathrm{GFP}\right)$. d-f, Translocation of the secreted products at forces of 0.5 , 1.0, and $2.0 \mu \mathrm{N}$ measured next to a single-neuron at DIV 6 with the corresponding density plots shown in g-i. d-f share the same time and current scale. i has a different x-axis scale. Violin and box plots with outliers (violin), mean (circle), median (bar), and whiskers of peak current shown in $\mathrm{j}$ and translocation time shown on a log scale in $\mathbf{k}$. l-m long-term measurement of a neurons' secretome at DIV 6 at a force setpoint of $1.0 \mu \mathrm{N}$ and a potential of $2 \mathrm{~V}$. The events are clustered in bundles of 68 minutes. I shows the density plot between the $0.55-0.95$ translocation time percentile. Arrows in $\mathbf{I}$ show the change of the median of the single clusters over time. UMAP dimension reduction of 5512 single events in $\mathbf{m}$ shows clockwise clustering according to experiment time. $\mathbf{n}$, increase of cell signaling over time as a result of event frequency change. 


\section{Experimental}

Experimental Setup: The experimental setup of the force controlled nanopore is based on the fluid force microscopy technology ${ }^{33}$ (FluidFM) which combines an atomic force microscope (AFM) and a micro-channeled cantilever with an apex at the tip. The force-controlled nanopore (FCNP) is formed at the interface between a soft PDMS substrate and a $\mathrm{Si}_{3} \mathrm{~N}_{4}$ micro-channeled AFM cantilever which is mounted on an Scanning Ion-Conductance Microscopy (SICM) setup as it was presented earlier ${ }^{56}$ and already has been used for the simultaneous measurement of topographical surface properties and surface charges on the nanoscale ${ }^{57}$ as well as for localized detection of ions and biomolecules with a forcecontrolled scanning nanopore microscope. ${ }^{39}$ On the AFM head (Nanosurf, Flex-Bio) a patch clamp amplifier (Tecella, Pico) is mounted which allows to connect the reservoir electrode to the amplifier and measure the ionic current between the reservoir and the reference electrodes (Figure S1a). In order to protect the setup for electromagnetic radiation, a Faraday ring is mounted on the AFM stage which furthermore fixes the sample dish (Figure S1b). The $\mathrm{Si}_{3} \mathrm{~N}_{4}$ cantilever (Cytosurge, nanopipette) is mounted on a holder that has a reservoir on the back side (Figure S1c). The nanopipette has an apex with a diameter of $300 \mathrm{~nm}$ at its tip (Figure S1d-e) and nominated spring constant of $2 \mathrm{~N} / \mathrm{m}$. To avoid cross contamination, each analyte was measured with a different probe and the experiments presented for each analyte were measured with the same probe.

For the calibration of the exact spring constant, the frequency spectrum and resonance peak of each single cantilever is used (Sader method ${ }^{58}$ ). At the edges of the apex, the cantilever has four sharp corners that form arches with a maximum height of 20-50 $\mathrm{nm}$ along the edges. The ionic current through the apex is measured by two silver chloride $(\mathrm{Ag} / \mathrm{AgCl})$ electrodes - a reference electrode in solution, outside of the cantilever and a working electrode in a reservoir connected to the micro-channel of the cantilever. The cantilever is mounted on an AFM head with a mounting angle of $\alpha=11^{\circ}$ allowing to control (Nanosurf, 
C3000) the force between the cantilever tip and the PDMS substrate. The approach of a cantilever in solution and at different force setpoints was observed though an optical prism $(0.3 \mathrm{~mm}$ right angle prism N-BK7, aluminum protected, Edmund optics) deflecting the light from the side view by $90^{\circ}$, into an optical microscope (Zeiss, Axiovert).

Fabrication of the PDMS substrate: The soft substrate was fabricated by spin-coating PDMS (Sylgard 184) at different curing agent ratios on a round microscope cover glass (diameter $24 \mathrm{~mm}$, thickness 0.13$0.16 \mathrm{~mm}$, ThermoScientific). Before curing, the PDMS was left at room temperature for five minutes to achieve a more homogeneous surface. Then the PDMS was cured in the oven at $80{ }^{\circ} \mathrm{C}$ or on a hotplate at $210^{\circ} \mathrm{C}$ for 120 min. The cured PDMS layer on glass was then glued into a dish chamber (Willco Wells).

Probe preparation and electrophysiological measurements: As a first step the micro-channeled cantilevers were plasma treated for 2 min (air plasma, at 18 W, using a PDC-32G; Harrick Plasma). Afterwards they were directly filled with $10 \mu \mathrm{L}$ of different buffer solutions $150 \mathrm{mM}$ phosphate-buffered saline (PBS, pH 7.4, Thermo Fisher, Gibco, 10010015), $2 \mathrm{M} \mathrm{KCl}$ at pH 8), or $100 \mathrm{mM}$ Hibernate $\mathrm{E}$ medium (Hib, pH $7.3 \pm 0.05$, Thermo Fisher, Gibco) and $0.5 \mu \mathrm{L}$ fluorescent dye (Lucifer yellow, Sigma Aldrich). For solution preparation ultrapure water (18.2 M $2 . \mathrm{cm}$, Milli-Q gradient A 10 from MerckMilliPore) was used. The solutions were then filtered with a $0.1 \mu \mathrm{m}$ filter (Merck, Millipore).

Next, the reservoir connector with the electrode and the connection to the pressure control (Figure S1c) was mounted on the backside reservoir of the probe and subsequently sealed with $90{ }^{\circ} \mathrm{C}$ warm paraffin wax. A potential bias was then set between the two electrodes and a pressure was applied to put the solution in the reservoir to the apex of the tip until a constant current resistance was reached. Leakage could be seen by strongly deviating resistances (Figure S2) or fluorescent liquid coming out of the probe other than the apex of the tip. 
Nanoparticle, DNA, peptide, and protein sensing: $8 \mu \mathrm{L}$ of 1\% solid concentrated carboxyl (-COOH) functionalized polystyrene nanobeads (PS20, Nanocs), with concentration of $8.86 \times 10^{14}$ particles per $\mathrm{mL}$, and a mean diameter of $20 \mathrm{~nm}$, were mixed with $4 \mathrm{~mL}$ filtered $\mathrm{KCl}$ buffer (10 mM Tris-HCl, $1 \mathrm{mM}$ EDTA, $0.15-2 \mathrm{M} \mathrm{KCl}$, pH 8.0). For smaller nanoparticles, $8 \mu \mathrm{L}$ of $8.86 \times 10^{14}$ particles per mL concentrated carboxyl (-COOH) functional gold particles (Aurion) with a mean diameter of $5.9 \pm 0.6 \mathrm{~nm}$ were mixed with 4 mL filtered $\mathrm{KCl}$ buffer.

Each $10 \mu \mathrm{g}$ NoLimits dsDNA fragments (Life Technologies Inc.) with 2000 and 20’000 base pairs were mixed in $4 \mathrm{~mL}$ filtered $\mathrm{KCl}$ buffer yielding a $1.92 \mathrm{nM}$ and $0.19 \mathrm{nM}$ solutions, respectively. The solutions were then filled into the PDMS coated dish (cis). A positive potential was applied inside the reservoir such that the DNA was dragged into the cantilever.

For peptide translocation measurements, porcine dynorphin A ( $\geq 95 \%$ (HPLC), $\geq 65$ wt\%, $2.15 \mathrm{kDa}$, Sigma-Aldrich) which has 17 residues (Tyr-Gly-Gly-Phe-Leu-Arg-Arg-Ile-Arg-Pro-Lys-Leu-Lys-TrpAsp-Asn-Gln) was used. $0.1 \mathrm{mg}$ (28.76 nmol) were dissolved in $100 \mu \mathrm{L}$ DI water (solubility checked on pepcalc.org) which yields a $0.29 \mathrm{mM}$ solution and stored in $10 \mu \mathrm{L}$ aliquots at $-20{ }^{\circ} \mathrm{C}$. Due to the positive charge of $+4 e^{-}$(pepcalc.org), the peptides were backfilled into the reservoir and electrophoretically driven out of the cantilever. First, the reservoir was filled with $10 \mu \mathrm{L}$ PBS and afterwards $1 \mu \mathrm{L}$ Dynorphin solution was mixed (26 $\mu \mathrm{M})$. Bovine Serum Albumin (BSA) and Laminin were purchased from Sigma Switzerland and solutions with $1 \mathrm{nM}$ concentration were prepared of which $4 \mathrm{~mL}$ were put into the Willco dish.

All, nanoparticles, DNA fragments, peptides and proteins were electrophoretically driven through the FCNP at applied electrical potentials between 0.01 to $2 \mathrm{~V}$. Data acquisition with the patch clamp amplifier was monitored with a customized LabView program (40 kHz sampling rate and a $10 \mathrm{kHz}$ digital filtering). 
A junction potential and a system capacitance was chosen to get a step function current response to a 200 ms long voltage step input (Figure S2). For data analysis a self-written code in Python 3 (available from the authors on request) was used.

\section{Antifouling modification of the PDMS substrate and cantilever for biomolecule and neuron}

studies: Other than nanoparticle, DNA, and neuron studies, for peptide and protein studies the PDMS substrate was coated with an anti-fouling protein resistant polymer PAcrAm-g-(PMOXA, $\mathrm{NH}_{2}, \mathrm{Si}$ ) in order to avoid sticking of biomolecules on the PDMS surface. In neuron studies this was not needed since culturing of the neurons on PDMS in the incubator for several days was sufficient to cover the PDMS completely with biomolecules. A detailed preparation of the polymers purchased from SuSoS AG Switzerland has been explained in following publications. ${ }^{59,60}$ The substrates were first plasma cleaned (100 W Oxygen plasma for $2 \mathrm{~min}$ ) and afterwards immersed in $0.1 \mathrm{mg} / \mathrm{mL}$ concentrated PMOXA solution with $1 \mathrm{mM}$ HEPES for $90 \mathrm{~min}$ at room temperature. Afterwards the substrate was rinsed three times with ultrapure water.

Same procedure was repeated for the $\mathrm{Si}_{3} \mathrm{~N}_{4}$ probes that were used for peptide, protein, and neuron measurements. When coating the cantilever, a slight under pressure was applied such that PMOXA coated also the inside of the pore opening during immersion.

Neuron culture: Cells were isolated from cerebral cortices of E18 embryos of time-mated pregnant Sprague Dawley rats (Janvier Labs). Approval for the animal experiments was obtained from the Cantonal Veterinary Office Zurich. The cortices were dissociated by incubating for $15 \mathrm{~min}$ in $5 \mathrm{~mL}$ of a filter sterilized solution of $0.5 \mathrm{mg} / \mathrm{mL}$ papain and $0.01 \mathrm{mg} / \mathrm{mL}$ deoxyribonuclease in PBS supplemented with 1 $\mathrm{mg} / \mathrm{mL}$ BSA and $10 \mathrm{mM}$ D-(+)-glucose in a $37^{\circ} \mathrm{C} / 5 \% \mathrm{CO}_{2}$ incubator. The supernatant was then removed and resuspended three times with $5 \mathrm{~mL}$ of Neurobasal medium (Thermo Fischer Scientific) supplemented 
with $2 \%$ GlutaMAX and $1 \%$ penicillin streptomycin. The remaining solution was gently triturated to break up the remaining tissue clumps. Cell solutions were spun down, re-suspended in fresh media and seeded on poly-d-lysine (Sigma-Aldrich) coated Wilco dishes (Wilco Wells) at a nominal seeding density of 100,000 neurons $/ \mathrm{cm}^{2}$ and incubated in a $37^{\circ} \mathrm{C} / 5 \% \mathrm{CO}_{2}$ incubator. Prior uncured, with PDMS coated glass dishes (see section 0) were glued on the glass slides of the Wilco dishes using a drop of uncured PDMS and subsequently cured for a minimum of two hours at $80^{\circ} \mathrm{C}$. Media was changed after $24 \mathrm{~h}$ to remove debris and dead cells and then weekly for the length of the experiment.

Measurements next to neurons: To monitor electrogenic activity of the neurons, the cells were incubated in Neurobasal medium with a $3 \mu \mathrm{M}$ concentration of the fluorescent $\mathrm{Ca}^{2+}$ indicator Fluo-4 AM (Thermo Fischer Scientific) for $1 \mathrm{~h}$, after which the medium was replaced with Neurobasal Hibernate $\mathrm{E}$ (Thermo Fischer Scientific) at $37{ }^{\circ} \mathrm{C}$. Hibernate $\mathrm{E}$ is a $\mathrm{CO}_{2}$ independent cell culture medium able to maintain embryonic neural cultures for up to 3 days. Background measurements in pure Hibernate $E$ are shown in Figure S13.

The dishes were then transferred to the FCNP setup which was surrounded by a self-built incubator box connected to a temperature controller (The Cube, Life Imaging Services, Switzerland). The cantilever was then placed with micro screws next to the neurons and a positive voltage inside the cantilever was applied at different voltages.

Data analysis: The structure of the code is shown in Figure S3. The slow drift of the baseline was corrected from the signal by using an Asymmetric Least Square (ALS) smoother ${ }^{61}$. It uses three parameters, a 2nd derivative constraint lambda normally set between $10^{3}$ and $10^{9}$, weighting of positive residuals normally between $10^{-3}$ and $10^{-9}$ and the amount of iterations which was always set to a value of 10. Since slow drift is removed from the signal every $\mathrm{n}^{\text {th }}$ point is used to calculate the drift where $\mathrm{n}$ is 
typically set between $10^{2}$ to $10^{4}$. The drift is subtracted from the signal and the mean of the drift is chosen as the baseline and added afterwards to the signal. In a second step the signal is filtered again with ASL smoother, this time building on the Whittaker smoother ${ }^{62}$ to smooth high frequencies. An intermediate threshold was defined using Otsu thresholding. ${ }^{63}$ It is assumed that there is more baseline signal than deviations from the baseline. Afterwards the standard deviations and means of the distributions above and below the Otsu threshold are used to redefine the baseline and event threshold.

$$
\text { normalized signal }=\frac{\text { signal }- \text { baseline }}{\mid \text { threshold }- \text { baseline } \mid}
$$

The signal is then normalized by the threshold and the baseline (equation 1). Subsequently, a data window (block) is shifted through the normalized signal and the block sum is computed which is used to define events in the signal. The block sum has the advantage that translocations are not automatically categorized as finished once the signal falls under the threshold but only if the data is under the threshold for a determined amount of time.

\section{Estimation of the nanopore size}

Equivalent cylindrical pore diameter: The analytical total resistance across a cylindrical nanopore is obtained by putting the resistances of the pore access, of the channel, and again of the pore access in series. It can be calculated using equation 2 where $\sigma$ is the bulk conductivity of the pore, $\mathrm{L}$ is the pore thickness, and $\mathrm{d}$ is the diameter of a perfectly round pore. ${ }^{64} \mathrm{~L}$ is set to $80 \mathrm{~nm}$ since this is the wall thickness of the cantilever.

$$
\frac{I}{V}=\frac{1}{R}=G=\sigma_{\text {bulk }}\left[\frac{4 L}{\pi d^{2}}+\frac{1}{d}\right]^{-1}
$$

The result of equation 2 is shown in Figure S4. In this first approach, a 2 M solution with a conductivity of $20.8 \mathrm{~S} / \mathrm{m}$ and an equivalent pore diameter of $300 \mathrm{~nm}$ yields a pore resistance of $0.3 \mathrm{M} \Omega$. In the approach 
experiments (Figure S9) the measured resistance under same conditions through the apex far away from the substrate is about $2 \mathrm{M} \Omega$. From equation 3 results that the combined resistance at the electrodes and through the bulk solution is about $1.7 \mathrm{M} \Omega$ in a $2 \mathrm{M}$ solution.

$$
R_{\text {bulk }}=R_{\text {total }}-R_{\text {pore }}
$$

The latter moreover implies that the pore resistance becomes the limiting factor once the probe is approached to the substrate. The nanopore resistance furthermore depends on the geometry, ${ }^{65}$ which however is neglected in this first approach but is taken into account in a 3D simulation of the ionic current through the apex of the cantilever.

3D simulation of ionic current through tip: A large micropore (300 nm in size) is pressed against a soft substrate (PDMS) at a mounting angle of $11^{\circ}$. An SEM illustrating the exact shape of the micropore is shown in Figure 1b. Subsequently, a nanoconfinement is created between the wall of the cantilever / micropore and the soft substrate. The nanoconfinement presumably has an oval shape (Figures S5 and S7). The nanopore is not round which is why the smallest dimension is defined as the pore opening. This dimension furthermore determines whether a biomolecule can pass through the pore or not.

Also the I-V curves in Figure 2a-b show ionic current rectification which is addressed to conically shaped nanopores. ${ }^{34,36,37}$ The size of the pore can be estimated by analyzing the ionic current through the pore at different solution concentrations and at different forces (Figure 2).

A finite element method simulation was implemented in COMSOL in order to estimate the nanopore size with respect to the ionic current. The nanopore size was defined as the largest distance between the edges of the apex (red lines in Figure S5) and the PDMS substrate, also called gap height $h_{g}(t)$. The tip was modeled by subtracting a bigger outer pyramid from an in inner smaller pyramid as depicted in Figure S5a. Afterwards a sphere with the center in the tip of the pyramid was subtracted from the remaining 
hollow pyramid structure to form an opening as this gets very close to 'corner lithography', the fabrication method used to fabricate the $300 \mathrm{~nm}$ opening at the tip of the cantilever. ${ }^{66}$ The simulation uses an electric currents solver to calculate the ionic current in the tip in series with an electrical circuit solver to account for the bulk resistance of $1.7 \mathrm{M} \Omega$ (for $2 \mathrm{M} \mathrm{KCl}$ solution) and the voltage source. Indentation into the substrate was modeled by slicing of the tip and taking the surface of the slice as bottom substrate. Substrate and $\mathrm{Si}_{3} \mathrm{~N}_{4}$ cantilever tip were modeled as electric insulator boundaries. The sides of the simulation volume, which is a cubic around the tip, were set to a constant ground potential of $0 \mathrm{~V}$. The bottom surface of the inner pyramid was connected to the electrical circuit using a terminal boundary.

Since the gap height $h_{g}(t)$ is a linear function of time $t$ with a constant approach speed at time zero $\left.v\right|_{t_{0}}$, related by the following equation $h_{g}(t)=h_{0}-h(t)=h_{0}-\left.\left(t-t_{0}\right) \cdot v\right|_{t_{0}}$, the x-axes Figure S6 and Figure 2c and $\mathbf{d}$ of the manuscript correspond to each other until the cantilever reaches the force set point and the gap height (i.e. nanopore size) stays constant. Simulation has been done for $2 \mathrm{M}$ and $0.1 \mathrm{M}$ solutions with conductivities of $20.683 \mathrm{~S} / \mathrm{m}$ and $1.672 \mathrm{~S} / \mathrm{m}$, respectively. Simulation with an angle of $11^{\circ}$ yielded circuit resistances as were measured during the experiments. Figure S7 shows the local electrical field in the pore at an applied potential of $1 \mathrm{~V}$ for both flat $\left(0^{\circ}\right)$ and $11^{\circ}$ indentation angle. 


\section{Supporting Information}

The Supporting Information is available free of charge on the ACS Publications website at DOI:

Experimental and data analysis, estimation of the nanopore size, FCNP formation on different PDMS

substrates, noise characterization and electrochemical measurements in different solutions, translocation

of nanoparticles and biomolecules, recording next to neurons (PDF).

\section{Corresponding Author}

* Email: Morteza Aramesh maramesh@ethz.ch

* Email: Tomaso Zambelli zambelli@biomed.ee.ethz.ch

\section{Acknowledgment}

We appreciate the technical assistance from S. Wheeler (ETH Zürich).

SNF, ETH Zurich and the FreeNovation grant are acknowledged for financial support.

D.M. is supported by the Swiss National Science Foundation Ambizione Grant (PZ00P2_174217/1).

M.A. is supported by Holcim Stiftung and the Career Seed Grant from ETH Zürich (project reference 020440-18) and the SPARK grant (PZ00P2_193242) from the Swiss National Science Foundation.

We thank Cytosurge AG, Switzerland for providing SEM images of different cantilever probes.

\section{Author contributions}

T.S., T.Z., J.V., and M.A. designed the experiments. T.S. performed the experiments. T.S. and D.M. did the COMSOL simulation. T.S. and C.F. performed the statistical analysis and coding. S.W. prepared the neuron cultures. All authors discussed the results and commented on the manuscript. T.S wrote the manuscript with support of T.Z. and M.A.

\section{Competing interests}

The authors declare no competing interests. 


\section{References}

(1) Alberts, B.; Johnson, A.; Lewis, J.; Raff, M.; Roberts, K.; Walter, P. Molecular Biology of the Cell. Garl. Sci. 2009.

(2) Cohen-Tanugi, D.; Lin, L. C.; Grossman, J. C. Multilayer Nanoporous Graphene Membranes for Water Desalination. Nano Lett. 2016, 16, 1027-1033.

(3) Guo, W.; Cao, L.; Xia, J.; Nie, F. Q.; Wen, M.; Xue, J.; Song, Y.; Zhu, D.; Wang, Y.; Jiang, L. Energy Harvesting with Single-Ion-Selective Nanopores: A Concentration-Gradient-Driven Nanofluidic Power Source. Adv. Funct. Mater. 2010, 20, 1339-1344.

(4) Taniguchi, M.; Ohshiro, T. Nanopore Device for Single-Molecule Sensing Method and Its Application. In Applications of Microfluidic Systems in Biology and Medicine; Tokeshi, M., Ed.; Springer Singapore: Singapore, 2019; pp. 301324.

(5) Clarke, J.; Wu, H. C.; Jayasinghe, L.; Patel, A.; Reid, S.; Bayley, H. Continuous Base Identification for Single-Molecule Nanopore DNA Sequencing. Nat. Nanotechnol. 2009, 4, 265-270.

(6) Jain, M.; Fiddes, I. T.; Miga, K. H.; Olsen, H. E.; Paten, B.; Akeson, M. Improved Data Analysis for the MinION Nanopore Sequencer. Nat. Methods 2015, 12, 351-356.

(7) Lu, H.; Giordano, F.; Ning, Z. Oxford Nanopore MinION Sequencing and Genome Assembly. Genomics. Proteomics Bioinformatics 2016, 14, 265-279.

(8) Pulcu, G. S. G. S.; Galenkamp, N. S.; Qing, Y.; Gasparini, G.; Mikhailova, E.; Matile, S.; Bayley, H. Single-Molecule Kinetics of Growth and Degradation of Cell-Penetrating Poly (Disulfide) S. J. Am. Chem. Soc. 2019, 141, 1244412447.

(9) Qing, Y.; Ionescu, S. A.; Bayley, H.; Pulcu, G. S.; Bayley, H. Directional Control of a Processive Molecular Hopper. Science 2018, 361, 908-912.

(10) Rodríguez-Manzo, J. A.; Puster, M.; Nicolaï, A.; Meunier, V.; Drndić, M. DNA Translocation in Nanometer Thick Silicon Nanopores. ACS Nano 2015, 9, 6555-6564.

(11) Merchant, C. A.; Healy, K.; Wanunu, M.; Ray, V.; Peterman, N.; Bartel, J.; Fischbein, M. D.; Venta, K.; Luo, Z.; Johnson, A. T. C.; et al. DNA Translocation through Graphene Nanopores. Nano Lett. 2010, 10, 2915-2921.

(12) Schneider, G. F.; Kowalczyk, S. W.; Calado, V. E.; Pandraud, G.; Zandbergen, H. W.; Vandersypen, L. M. K.; Dekker, C. DNA Translocation through Graphene Nanopores. Nano Lett. 2010, 10, 3163-3167.

(13) Liu, K.; Feng, J.; Kis, A.; Radenovic, A. Atomically Thin Molybdenum Disulfide Nanopores with High Sensitivity for Dna Translocation. ACS Nano 2014, 8, 2504-2511.

(14) Danda, G.; Masih Das, P.; Chou, Y. C.; Mlack, J. T.; Parkin, W. M.; Naylor, C. H.; Fujisawa, K.; Zhang, T.; Fulton, L. B.; Terrones, M.; et al. Monolayer WS2Nanopores for DNA Translocation with Light-Adjustable Sizes. ACS Nano 2017, 11, 1937-1945.

(15) Freedman, K. J.; Otto, L. M.; Ivanov, A. P.; Barik, A.; Oh, S.-H. H.; Edel, J. B. Nanopore Sensing at Ultra-Low Concentrations Using Single-Molecule Dielectrophoretic Trapping. Nat. Commun. 2016, 7, 1-9.

(16) Bell, N. A. W.; Keyser, U. F. Digitally Encoded DNA Nanostructures for Multiplexed, Single-Molecule Protein Sensing with Nanopores. Nat. Nanotechnol. 2016, 11, 645-651.

(17) Robertson, J. W. F. F.; Reiner, J. E. The Utility of Nanopore Technology for Protein and Peptide Sensing. Proteomics 2018, 18, 1800026.

(18) Freedman, K. J.; Haq, S. R.; Edel, J. B.; Jemth, P.; Kim, M. J. Single Molecule Unfolding and Stretching of Protein Domains inside a Solid-State Nanopore by Electric Field. Sci. Rep. 2013, 3, 1638. 
(19) Payet, L.; Martinho, M.; Pastoriza-Gallego, M.; Betton, J. M.; Auvray, L.; Pelta, J.; Mathé, J. Thermal Unfolding of Proteins Probed at the Single Molecule Level Using Nanopores. Anal. Chem. 2012, 84, 4071-4076.

(20) Restrepo-Pérez, L.; John, S.; Aksimentiev, A.; Joo, C.; Dekker, C. SDS-Assisted Protein Transport through Solid-State Nanopores. Nanoscale 2017, 9, 11685-11693.

(21) Yusko, E. C.; Johnson, J. M.; Majd, S.; Prangkio, P.; Rollings, R. C.; Li, J.; Yang, J.; Mayer, M. Controlling Protein Translocation through Nanopores with Bio-Inspired Fluid Walls. Nat. Nanotechnol. 2011, 6, 253-260.

(22) Eggenberger, O. M.; Ying, C.; Mayer, M. Surface Coatings for Solid-State Nanopores. Nanoscale 2019, 11, 1963619657.

(23) Awasthi, S.; Sriboonpeng, P.; Ying, C.; Houghtaling, J.; Shorubalko, I.; Marion, S.; Davis, S. J.; Sola, L.; Chiari, M.; Radenovic, A. Polymer Coatings to Minimize Protein Adsorption in Solid

Ssitate Methowlored20, 2000177.

(24) Lee, K.; Park, K. B.; Kim, H. J. M. J. M. H.; Yu, J. S.; Chae, H.; Kim, H. J. M. J. M. H.; Kim, K. B. Recent Progress in Solid-State Nanopores. Adv. Mater. 2018, 30, 1704680.

(25) Shekar, S.; Niedzwiecki, D. J.; Chien, C. C.; Ong, P.; Fleischer, D. A.; Lin, J.; Rosenstein, J. K.; Drndić, M.; Shepard, K. L. Measurement of DNA Translocation Dynamics in a Solid-State Nanopore at 100 Ns Temporal Resolution. Nano Lett. 2016, 16, 4483-4489.

(26) Pud, S.; Chao, S. H.; Belkin, M.; Verschueren, D.; Huijben, T.; Van Engelenburg, C.; Dekker, C.; Aksimentiev, A. Mechanical Trapping of DNA in a Double-Nanopore System. Nano Lett. 2016, 16, 8021-8028.

(27) Cadinu, P.; Paulose Nadappuram, B.; Lee, D. J.; Sze, J. Y. Y.; Campolo, G.; Zhang, Y.; Shevchuk, A.; Ladame, S.; Albrecht, T.; Korchev, Y.; et al. Single Molecule Trapping and Sensing Using Dual Nanopores Separated by a Zeptoliter Nanobridge. Nano Lett. 2017, 17, 6376-6384.

(28) Al Sulaiman, D.; Cadinu, P.; Ivanov, A. P.; Edel, J. B.; Ladame, S. Chemically Modified Hydrogel-Filled Nanopores: A Tunable Platform for Single-Molecule Sensing. Nano Lett. 2018, 18, 6084-6093.

(29) Zhao, Y.; Xie, W.; Tian, E.; Ren, Y.; Zhu, J.; Deng, Y.; He, S.; Liang, L.; Wang, Y.; Zhou, D.; et al. Slowing down DNA Translocation by a Nanofiber Meshed Layer. J. Phys. D. Appl. Phys. 2018, 51, 45402.

(30) Fologea, D.; Uplinger, J.; Thomas, B.; McNabb, D. S.; Li, J. Slowing DNA Translocation in a Solid-State Nanopore. Nano Lett. 2005, 5, 1734-1737.

(31) Lu, B.; Hoogerheide, D. P.; Zhao, Q.; Zhang, H.; Tang, Z.; Yu, D.; Golovchenko, J. A. Pressure-Controlled Motion of Single Polymers through Solid-State Nanopores. Nano Lett. 2013, 13, 3048-3052.

(32) Karmi, A.; Sakala, G. P.; Rotem, D.; Reches, M.; Porath, D. Durable, Stable, and Functional Nanopores Decorated by Self- Assembled Dipeptides. 2020, 3-8.

(33) Meister, A. A.; Gabi, M.; Behr, P.; Studer, P.; Vörös, J.; Niedermann, P.; Bitterli, J.; Polesel-Maris, J. J.; Liley, M.; Heinzelmann, H.; et al. FluidFM: Combining Atomic Force Microscopy and Nano Uidics in a Universal Liquid Delivery System for Single Cell Applications and Beyond. Nano Lett. 2009, 9, 2501-2507.

(34) Siwy, Z. S. Ion-Current Rectification in Nanopores and Nanotubes with Broken Symmetry. Adv. Funct. Mater. 2006, 16, 735-746.

(35) Sa, N.; Baker, L. A. Rectification of Nanopores at Surfaces. J. Am. Chem. Soc. 2011, 133, 10398-10401.

(36) Sa, N.; Lan, W.-J.; Shi, W.; Baker, L. A. Rectification of Ion Current in Nanopipettes by External Substrates. ACS Nano 2013, 7, 11272-11282.

(37) Zeng, S.; Wen, C.; Solomon, P.; Zhang, S. L.; Zhang, Z. Rectification of Protein Translocation in Truncated Pyramidal Nanopores. Nat. Nanotechnol. 2019, 14, 1056-1062. 
(38) Smeets, R. M. M.; Keyser, U. F.; Krapf, D.; Wu, M.-Y.; Dekker, N. H.; Dekker, C. Salt Dependence of Ion Transport and DNA Translocation through Solid-State Nanopores. Nano Lett. 2006, 6, 89-95.

(39) Aramesh, M.; Forró, C.; Dorwling-Carter, L.; Lüchtefeld, I.; Schlotter, T.; Ihle, S. J.; Shorubalko, I.; Hosseini, V.; Momotenko, D.; Zambelli, T.; et al. Localized Detection of Ions and Biomolecules with a Force-Controlled Scanning Nanopore Microscope. Nat. Nanotechnol. 2019, 14, 791-798.

(40) Feng, J.; Liu, K.; Bulushev, R. D.; Khlybov, S.; Dumcenco, D.; Kis, A.; Radenovic, A. Identification of Single Nucleotides in MoS 2 Nanopores. Nat. Nanotechnol. 2015, 10, 1070-1076.

(41) Plesa, C.; Verschueren, D.; Pud, S.; Van Der Torre, J.; Ruitenberg, J. W.; Witteveen, M. J.; Jonsson, M. P.; Grosberg, A. Y.; Rabin, Y.; Dekker, C. Direct Observation of DNA Knots Using a Solid-State Nanopore. Nat. Nanotechnol. 2016, 11, 1093-1097.

(42) Briggs, K.; Madejski, G.; Magill, M.; Kastritis, K.; De Haan, H. W.; McGrath, J. L.; Tabard-Cossa, V. DNA Translocations through Nanopores under Nanoscale Preconfinement. Nano Lett. 2018, 18, 660-668.

(43) Knoll, A. T.; Carlezon, W. A. Dynorphin, Stress, and Depression. Brain Res. 2010, 1314, 56-73.

(44) Chen, H.; Li, L.; Zhang, T.; Qiao, Z.; Tang, J.; Zhou, J. Protein Translocation through a MoS2 Nanopore:A Molecular Dynamics Study. J. Phys. Chem. C 2018, 122, 2070-2080.

(45) Luckenbill-Edds, L. Laminin and the Mechanism of Neuronal Outgrowth. Brain Res. Rev. 1997, 23, 1-27.

(46) Böhme, U.; Scheler, U. Effective Charge of Bovine Serum Albumin Determined by Electrophoresis NMR. Chem. Phys. Lett. 2007, 435, 342-345.

(47) Majorek, K. A.; Porebski, P. J.; Dayal, A.; Zimmerman, M. D.; Jablonska, K.; Stewart, A. J.; Chruszcz, M.; Minor, W. Structural and Immunologic Characterization of Bovine, Horse, and Rabbit Serum Albumins. Mol. Immunol. 2012, 52, 174-182.

(48) Li, J.; Fologea, D.; Rollings, R.; Ledden, B. Characterization of Protein Unfolding with Solid-State Nanopores. Protein Pept. Lett. 2014, 21, 256-265.

(49) Gu, C.; Yu, Z.; Li, X.; Zhu, X.; Cao, Z.; Ye, Z.; Jin, C.; Liu, Y. Experimental Study of Protein Translocation through MoS2 Nanopores. Appl. Phys. Lett. 2019, 115, 1-6.

(50) Forró, C.; Thompson-Steckel, G.; Weaver, S.; Weydert, S.; Ihle, S.; Dermutz, H.; Aebersold, M. J.; Pilz, R.; Demkó, L.; Vörös, J. Modular Microstructure Design to Build Neuronal Networks of Defined Functional Connectivity. Biosens. Bioelectron. 2018, 122, 75-87.

(51) Aebersold, M. J.; Dermutz, H.; Demkó, L.; Cogollo, J. F. S.; Lin, S.; Burchert, C.; Schneider, M.; Ling, D.; Forró, C.; Han, H. Local Chemical Stimulation of Neurons with the Fluidic Force Microscope (FluidFM). ChemPhysChem 2018, 19, 1234-1244.

(52) Levitan, I. B.; Kaczmarek, L. K. The Neuron: Cell and Molecular Biology; Oxford University Press, USA, 2015.

(53) Janas, A. M.; Sapoń, K.; Janas, T.; Stowell, M. H. B.; Janas, T. Exosomes and Other Extracellular Vesicles in Neural Cells and Neurodegenerative Diseases. Biochim. Biophys. Acta - Biomembr. 2016, 1858, 1139-1151.

(54) McInnes, L.; Healy, J.; Melville, J. Umap: Uniform Manifold Approximation and Projection for Dimension Reduction. arXiv Prepr. arXiv1802.03426 2018.

(55) Becht, E.; McInnes, L.; Healy, J.; Dutertre, C.-A.; Kwok, I. W. H.; Ng, L. G.; Ginhoux, F.; Newell, E. W. Dimensionality Reduction for Visualizing Single-Cell Data Using UMAP. Nat. Biotechnol. 2019, 37, 38.

(56) Ossola, D.; Dorwling-Carter, L.; Dermutz, H.; Behr, P.; Vörös, J.; Zambelli, T. Simultaneous Scanning Ion Conductance Microscopy and Atomic Force Microscopy with Microchanneled Cantilevers. Phys. Rev. Lett. 2015, 115, 238103. 
(57) Dorwling-Carter, L.; Aramesh, M.; Han, H.; Zambelli, T.; Momotenko, D. Combined Ion Conductance and Atomic Force Microscope for Fast Simultaneous Topographical and Surface Charge Imaging. Anal. Chem. 2018, 90, 1145311460.

(58) Sader, J. E.; Larson, I.; Mulvaney, P.; White, L. R. Method for the Calibration of Atomic Force Microscope Cantilevers. Rev. Sci. Instrum. 1995, 66, 3789-3798.

(59) Serrano, Â.; Zürcher, S.; Tosatti, S.; Spencer, N. D. Imparting Nonfouling Properties to Chemically Distinct Surfaces with a Single Adsorbing Polymer: A Multimodal Binding Approach. Macromol. Rapid Commun. 2016, 37, 622-629.

(60) Weydert, S.; Zürcher, S.; Tanner, S.; Zhang, N.; Ritter, R.; Peter, T.; Aebersold, M. J.; Thompson-Steckel, G.; Forró, C.; Rottmar, M. Easy to Apply Polyoxazoline-Based Coating for Precise and Long-Term Control of Neural Patterns. Langmuir 2017, 33, 8594-8605.

(61) Eilers, P. H. C.; Boelens, H. F. M. Baseline Correction with Asymmetric Least Squares Smoothing. Leiden Univ. Med. Cent. Rep. 2005, 1, 5.

(62) Eilers, P. H. C. A Perfect Smoother. Anal. Chem. 2003, 75, 3631-3636.

(63) Otsu, N. A Threshold Selection Method from Gray-Level Histograms. IEEE Trans. Syst. Man. Cybern. 1979, 9, 6266.

(64) Kowalczyk, S. W.; Grosberg, A. Y.; Rabin, Y.; Dekker, C. Modeling the Conductance and DNA Blockade of SolidState Nanopores. Nanotechnology 2011, 22, 315101.

(65) Liu, K.; Lihter, M.; Sarathy, A.; Caneva, S.; Qiu, H.; Deiana, D.; Tileli, V.; Alexander, D. T. L.; Hofmann, S.; Dumcenco, D.; et al. Geometrical Effect in 2D Nanopores. Nano Lett. 2017, 17, 4223-4230.

(66) Berenschot, E.; Tas, N. R.; Jansen, H. V; Elwenspoek, M. 3D-Nanomachining Using Corner Lithography. In 2008 3rd IEEE International Conference on Nano/Micro Engineered and Molecular Systems; IEEE, 2008; pp. 729-732. 